\title{
Intracranial complications arising from acute otitis media / mastoiditis - 10 year tertiary centre experience
}

\author{
Daniel Moualed, John Curran
}

John Radcliffe Hospital, Oxford

Background: Intra-cranial complications of acute otitis media or mastoiditis are rare but can result in significant morbidity and death.

Objectives: To determine the range of presenting features and clinical course of adults with intra-cranial complications of suppurative ear disease.

Methods: Retrospective case note review was performed to collect demographic, clinical, and radiological data.

Results:

Demographics - 41 patients with mean age 48.4 (range 2177) were admitted to our institution from 2007 to 2017.

Complications - The intracranial complications included: venous sinus thrombosis $(n=3)$, meningitis/encephalitis $(n=20)$, extra-dural abscess $(n=2)$, sub-dural abscess/empyema $(n=8)$, temporal lobe abscess $(n=4)$, and posterior fossa abscess $(n=4)$.

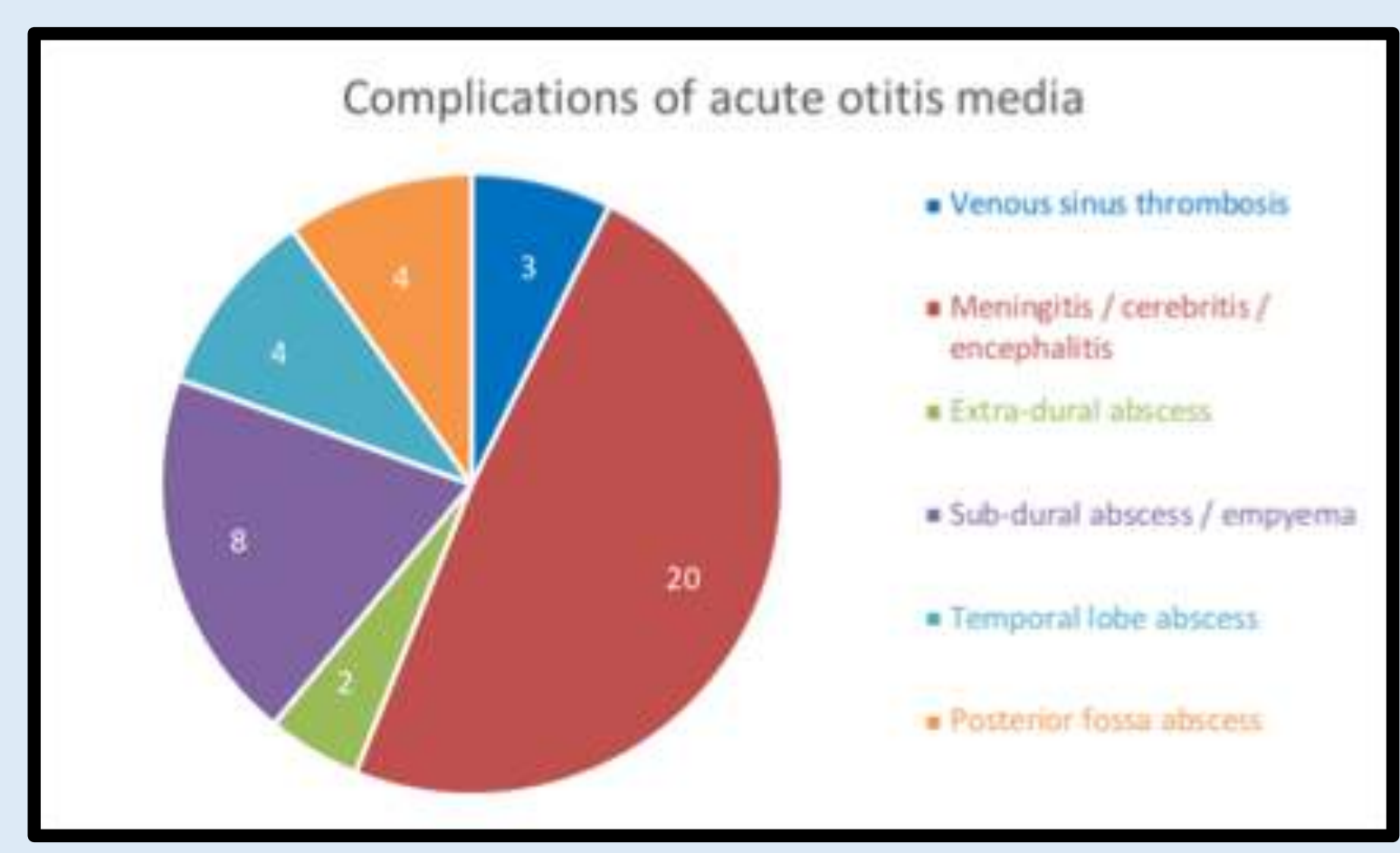

ENT surgical intervention - 10 patients did not have otological surgery (mostly cases of meningitis and good early response to antibiotics) 10 had grommet insertion, 21 had mastoidectomy. 3 patients initially treated with myringotomy and grommet insertion subsequently underwent mastoidectomy.

6 patients had cholesteatoma, of which 4 presented with posterior fossa abscess. No patients presented with posterior fossa collection without cholesteatoma.
Neurosurgical intervention - 16 patients required neurosurgical intervention including: ICP monitor insertion $(n=3)$, EVD insertion $(n=6)$, burr-hole drainage of abscess $(n=4)$, craniotomy $(n=6)$

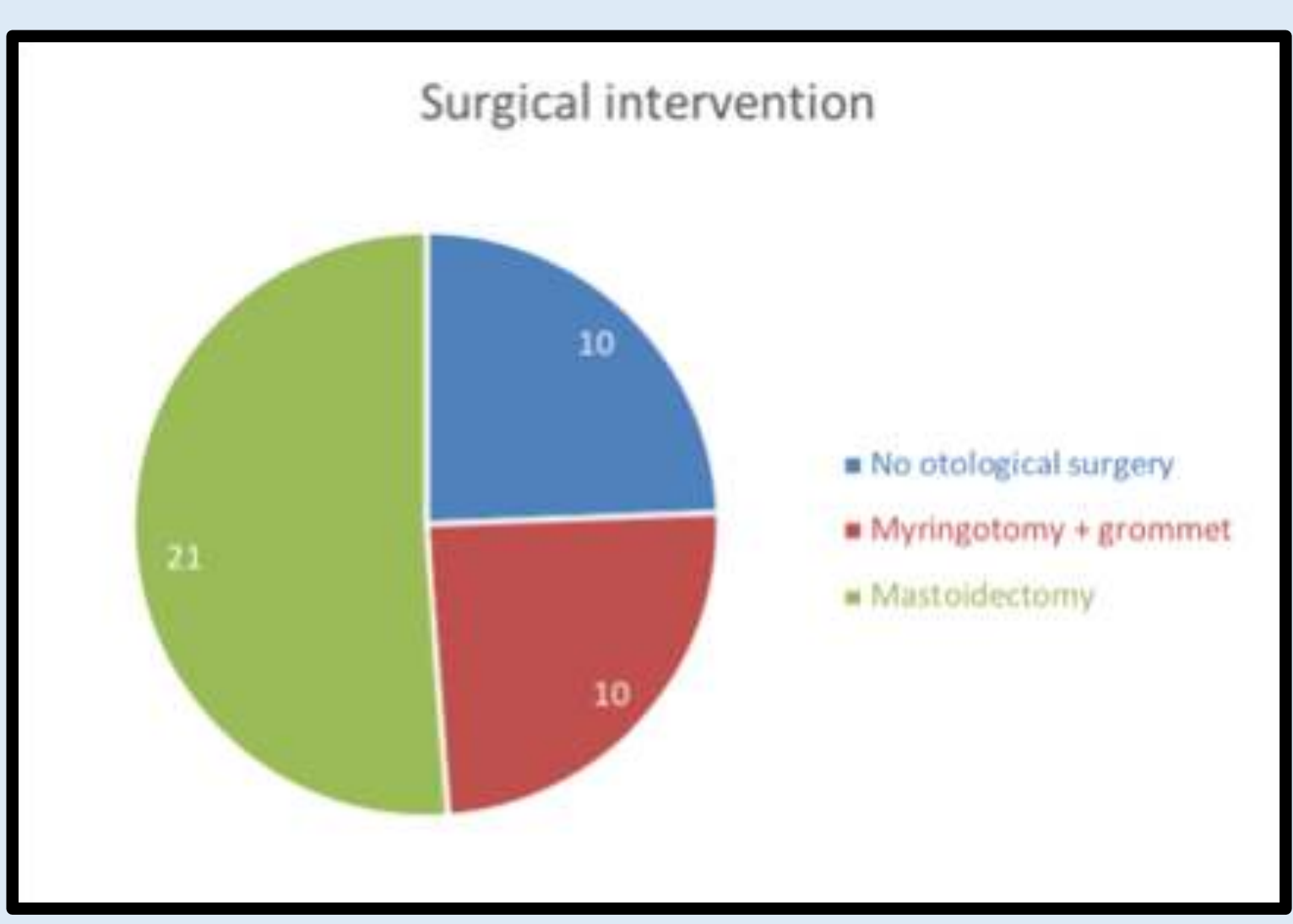

The total length of stay ranged from 1-154 days with a mean hospital stay of 30 days. Long-term morbidity was primarily due to neurological complications including epilepsy and hemiparesis.

4 Patients in our series died, there was no identifiable predictive factor that correlated with fatal outcome.

\begin{tabular}{|l|l|}
\hline Significant long-term morbidity / death & $\mathbf{n}=$ \\
\hline Epilepsy & 1 \\
\hline Hemiparesis & 6 \\
\hline Death & 4 \\
\hline
\end{tabular}

Conclusions: Intra-cranial complications of AOM affect a wide age range and present most commonly with meningitis / encephalitis. Over one third will require neurosurgical intervention.

\section{A majority of patients recovered fully, however a significant number suffered long-term neurological morbidity and 4 patients died.}

Cholesteatoma was strongly associated with posterior fossa abscess formation in our series. 